\title{
ORIGINAL
}

\section{COSTE DE LA DIARREA ASOCIADA A CLOSTRIDIUM DIFFICILE EN ESPAÑA}

\author{
Ángel Asensio (1), Emilio Bouza (2), Santiago Grau (3), Darío Rubio-Rodríguez (4), Carlos Rubio- \\ Terrés (4).
}

(1) Servicio de Medicina Preventiva, Hospital Universitario Puerta de Hierro, Majadahonda, Madrid.

(2) Servicio de Microbiología y Enfermedades Infecciosas, Hospital General Universitario Gregorio Marañón, Madrid.

(3) Servicio de Farmacia, Hospital del Mar, Barcelona.

(4) Health Value. Madrid.

\begin{abstract}
RESUMEN
Fundamentos: No se dispone de estudios adecuados del coste de las diarreas asociadas a Clostridium difficile (DACD) en España. El objetivo del estudio es estimar el coste de las DACD para el Sistema Nacional de Salud (SNS).

Métodos: Se realizó un modelo económico para calcular el coste por episodio de DACD (por antibióticos, prolongación del ingreso hospitalario, procedimientos quirúrgicos, medidas de control de la infección, recurrencias de las infecciones tratadas) y el coste anual de la DACD. El uso de recursos en la práctica clínica se obtuvo mediante un panel Delphi de clínicos españoles con experiencia en la DACD y los costes unitarios ( $€$ 2012) de fuentes españolas.

Resultados: Se estimó que anualmente se produjeron 7.601 episodios de DACD en España (incidencia de 17,1 episodios/año/10.000 altas hospitalarias) con un gasto anual para el SNS de 32.157.093 $€$. Coste por episodio de DACD: $3.901 €$ (infección inicial), $4.875 €$ (primera recurrencia) y $5.916 €$ (segunda recurrencia). Coste total de las recurrencias: $10.426 .750 €$ anuales. El $95,6 \%$ del gasto se debería a la prolongación de la duración de la estancia hospitalaria, el $0,5 \%$ a tratamientos antibióticos, el 2,8\% a intervenciones quirúrgicas y el 1,1\% a las medidas de control de la infección. Los resultados del estudio fueron sensibles a la incidencia y tasa de letalidad de la DACD en España y a la duración de la prolongación de la duración de la estancia hospitalaria debida a la DACD
\end{abstract}

Conclusiones: Según este estudio, el gasto asociado a la DACD se debe fundamentalmente a la prolongación de los ingresos hospitalarios y tiene un gran impacto económico en el SNS.

Palabras clave: Clostridium difficile. Diarrea. Vancomicina. Metronidazol. Coste de la enfermedad.

\section{ABSTRACT Cost of Clostridium difficile Associated Diarrhea in Spain}

Background: There are not available adequate studies of the costs of Clostridium difficile-associated diarrhea (CDAD) in Spain. The aim of the study is to estimate the cost of CDAD for the National Health Service (NHS).

Methods: an economic model was carried out to calculate the cost per episode of CDAD (due to antimicrobials, prolonged hospitalization, surgical procedures, measures to control the infection, recurrences of infections treated) and the CDAD annual cost. Resources use in clinical practice was obtained through a Delphi panel of Spanish clinicians with expertise in CDAD and unit costs ( $€$ 2012) from Spanish Sources.

Results: An estimated 7,601 episodes of CDAD occur annually in Spain (incidence of 17.1 episodes/year/10,000 hospital discharges) with an annual cost to the NHS of $€ 32,157,093$. Cost per episode of CDAD: $€ 3,901$ (initial infection), $€ 4,875$ (first recurrence) and $€ 5,916$ (second recurrence). Total cost of recurrences: $€ 10,426,750$ annually. The $95.6 \%$ of spending is due to the prolonged hospitalization, $0.5 \%$ to antibiotic treatment, $2.8 \%$ for surgery and $1.1 \%$ for measures of control infection. Study results are sensitive to incidence and case fatality rate of CDAD in Spain and the prolongation of duration of hospital stay due to CDAD

Conclusions: According to this study, the expense associated with CDAD is primarily due to the prolongation of hospitalization and has a great economic impact on the NHS.

Key words: Clostridium difficile. Diarrhea. Vancomicin. Metronidazole. Cost of illness. 


\section{INTRODUCCIÓN}

Clostridium difficile es un microorganismo capaz de proliferar en la luz intestinal y producir toxinas, siendo la causa más frecuente de diarrea nosocomial $(\mathrm{DACD})^{1}$. Este microorganismo es causante de un espectro de enfermedades denominadas infecciones por Clostridium difficile (ICD), que va desde un cuadro de diarrea no complicada y evolución benigna hasta cuadros de progresiva gravedad que incluyen la colitis pseudomembranosa y el megacolon tóxico ${ }^{2}$. La incidencia anual de la DACD en España se estima en 17,1 casos por cada 10.000 pacientes hospitalizados $^{3}$ oscilando entre 12,2 y 24,0 casos por cada 10.000 altas e ingresos hospitalarios, respectivamente ${ }^{4,5}$. En un reciente estudio español, la letalidad hospitalaria fue muy superior en los casos con DACD (31\%) en comparación con los controles que no presentaban esta infección $(6,6 \%)^{6}$.

En la actualidad, dependiendo de la gravedad del proceso, el tratamiento de elección de la DACD es metronidazol o vancomicina ${ }^{1}$. Sin embargo, de acuerdo con un metaanálisis publicado en $2012^{7} \mathrm{el}$ tratamiento de la DACD con metronidazol y vancomicina fracasa en el $22,4 \%$ y el $14,2 \%$ de los casos, respectivamente, y se produce recurrencia de la infección después del tratamiento en el $27,1 \%$ y el $24,0 \%$, respectivamente. Una recurrencia suele venir seguida por episodios repetidos en el $65 \%$ de los casos, lo que conlleva considerables gastos hospitalarios asociados a esta infección ${ }^{8}$.

Se dispone de pocos estudios publicados que intentaron estimar el consumo de recursos asociados a la DACD en Espa$\tilde{n} a^{6,9}$. Sin embargo, no discriminaron el consumo de recursos realmente debidos a la DACD, separándolos de los originados por la enfermedad de base. Por lo que respecta a otros países europeos, según una reciente revisión sistemática ${ }^{10}$ solo se dispone de datos de dos países (Irlanda y Alemania), estimándose que el coste incremental por caso de ICD (en euros del año 2012) ascendería aproximadamente a $5.600 €$ en Irlanda y $13.900 €$ en Alemania. Estos datos indican que la DACD tiene un considerable impacto económico.

El objetivo del presente estudio fue estimar el coste de las DACD para el Sistema Nacional de Salud (SNS).

\section{MATERIAL Y MÉTODOS}

Desde la perspectiva del SNS, mediante un modelo económico se analizó el coste actual de la DACD en los pacientes adultos (a partir de los 18 años de edad), tanto a nivel nacional como autonómico, tratados durante un año con metronidazol $\mathrm{y}$ vancomicina.

El coste anual de la enfermedad ( $€$ de 2012) se estimó a partir de los datos de incidencia, altas hospitalarias y letalidad publicados en España ${ }^{3-6,11}$ y se restringió a los costes producidos por los episodios de DACD nosocomiales (excluyéndose los costes originados por la enfermedad de base). Se consideró que en España se producen 4.720 .545 altas hospitalarias al año ${ }^{11}$, que la incidencia anual media de la DACD es de 17,1 casos (12,2-24,0) por cada 10.000 altas hospitalarias ${ }^{3-5}$ y una tasa de letalidad global en los casos de DACD del $31 \% \%^{6}$, oscilando entre el $12,3 \%$ y el $48,0 \%{ }^{4,6}$. Estas tasas de letalidad (debidas a todas las causas: la enfermedad de base, la DACD y demás) se utilizaron para calcular el número neto de pacientes con DACD (tabla 1). El cálculo del coste de la enfermedad para cada Comunidad Autónoma se hizo según sus pesos poblacionales.

Las estimaciones sobre las tasas de utilización de los tratamientos actuales (metronidazol y vancomicina), tanto en la 
Tabla 1

Premisas adoptadas en el análisis del coste de la DACD

\begin{tabular}{|c|c|c|c|c|}
\hline Ítem & Caso base* & Mínima & Máxima & Referencias \\
\hline \multicolumn{5}{|l|}{ Datos poblacionales } \\
\hline Altas hospitalarias en España (año 2.010) & 4.720 .545 & 4.720 .545 & 4.720 .545 & 11 \\
\hline Incidencia anual de DACD (por $10^{4}$ altas hosp.) & 17,1 & 12,2 & 24 & 3,5 \\
\hline Tasa de letalidad en los pacientes con DACD & $31,0 \%$ & $12,3 \%$ & $48,0 \%$ & 4,6 \\
\hline \multicolumn{5}{|l|}{ Tratamientos de la $\mathrm{DACD}$} \\
\hline Antibióticos & Metronidazol oral & Vancomicina oral & & \\
\hline Infección inicial & $68 \%$ & $32 \%$ & & $\mathrm{PD}$ \\
\hline $1^{\mathrm{a}}$ recurrencia & $44 \%$ & $56 \%$ & & PD \\
\hline $2^{\mathrm{a}}$ recurrencia & $33 \%$ & $67 \%$ & & PD \\
\hline \multicolumn{5}{|l|}{ Tasas de recurrencia de los tratamientos de la $D A C D$} \\
\hline Recurrencia & Metronidazol oral & Vancomicina oral & & \\
\hline Infección inicial & $29,0 \%$ & $24,0 \%$ & & 12,13 \\
\hline $1^{\mathrm{a}}$ recurrencia & $30,0 \%$ & $35,5 \%$ & & 14,15 \\
\hline \multicolumn{5}{|l|}{ Uso de recursos sanitarios } \\
\hline \multicolumn{5}{|l|}{ Número de estancias hospitalarias } \\
\hline Infección inicial & 7,4 & 3,7 & 8 & $\mathrm{PD}$ \\
\hline $1^{\mathrm{a}}$ recurrencia & 9,1 & 5 & 9,7 & PD \\
\hline $2^{\mathrm{a}}$ recurrencia & 10,8 & 7,3 & 11,3 & PD \\
\hline \multicolumn{5}{|l|}{ Tasa de ingreso en UCI } \\
\hline Infección inicial & $9,6 \%$ & $0,3 \%$ & $11,0 \%$ & PD \\
\hline $1^{\mathrm{a}}$ recurrencia & $11,7 \%$ & $1,0 \%$ & $13,3 \%$ & PD \\
\hline $2^{\mathrm{a}}$ recurrencia & $13,1 \%$ & $1,0 \%$ & $15,0 \%$ & PD \\
\hline Tasa de intervención quirúrgica & $1,2 \%$ & $0,3 \%$ & $1,3 \%$ & PD \\
\hline \multicolumn{5}{|l|}{ Medidas de control de la infección (por episodio) } \\
\hline $\mathrm{N}^{\mathrm{o}}$ de guantes desechables & 35 & 12 & 50 & $\mathrm{PD}$ \\
\hline $\mathrm{N}^{\mathrm{o}}$ de batas desechables & 25 & 6 & 25 & $\mathrm{PD}$ \\
\hline $\mathrm{N}^{\mathrm{o}}$ de termómetros desechables & 30 & 3 & 50 & PD \\
\hline \multicolumn{5}{|l|}{ Costes (€ de 2012) } \\
\hline Metronidazol (20 unidades de $250 \mathrm{mg}$ )* & $1,08 €$ & - & - & 19 \\
\hline Vancomicina (1 unidad de $125 \mathrm{mg}$ )* & $3,45 €$ & - & - & 19 \\
\hline Día de ingreso hospitalario & $434,00 €$ & $390,60 €$ & $477,40 €$ & 16 \\
\hline Día de ingreso en UCI & $1.131,07 €$ & $1.017,96 €$ & $1.244,18 €$ & 16 \\
\hline Intervención quirúrgica (GRD 148 y 149) & $9.868,83 €$ & $7.561,89 €$ & $12.175,78 €$ & 16 \\
\hline Guante desechable (unidad) & $0,03 €$ & $0,03 €$ & $0,03 €$ & 20 \\
\hline Bata desechable (unidad) & $0,22 €$ & $0,20 €$ & $0,24 €$ & 21 \\
\hline Termómetro desechable (unidad) & $0,1548 €$ & $0,1393 €$ & $0,1703 €$ & 22 \\
\hline
\end{tabular}

DACD: diarrea asociada a Clostridium difficile; GRD: grupo relacionado por el diagnóstico; PD: Panel Delphi; PVL: precio de venta del laboratorio; UCI: Unidad de cuidados intensivos. *PVL con 7,5\% de descuento. 
infección inicial como en el caso de producirse una primera o una segunda recurrencia, se realizaron mediante un panel Delphi de 3 expertos clínicos (dos infectólogos y un farmacéutico de hospital) con una amplia experiencia en el manejo de la DACD (tabla 1). Se llegó al consenso después de una reunión presencial y la respuesta a un cuestionario mediante dos rondas. Los tres expertos clínicos son coautores del presente trabajo.

Las tasas de recurrencia de la infección con metronidazol y vancomicina se obtuvieron de los datos publicados, tanto en la infección inicial ${ }^{12,13}$ como después del tratamiento de la primera recurrencia ${ }^{14,15}$ (tabla 1).

El uso de recursos sanitarios (número de estancias hospitalarias, probabilidad de ingreso en UCI, posibilidad de intervención quirúrgica (colectomía y hemicolectomía) y medidas de control de la infección como los guantes, batas y termómetros desechables) se estimaron mediante el panel de expertos ya mencionado (tabla 1).

Todos los costes unitarios se obtuvieron de fuentes españolas ${ }^{16-22}$ (tabla 1). El coste medio de un día de estancia hospitalaria, en planta o en UCI, así como el coste de las intervenciones quirúrgicas (promedio de los GRD 148 y 149) se obtuvo de los precios públicos de las $\mathrm{CCAA}^{16}$. El coste total por paciente de los diferentes tratamientos antibióticos (precios de venta al laboratorio [PVL] con el 7,5\% de descuento) se calculó para las pautas posológicas recomendadas (metronidazol oral $1.500 \mathrm{mg} /$ día; vancomicina oral: 500 $\mathrm{mg} /$ día en la infección inicial, 1.000 $\mathrm{mg} /$ día en la primera recurrencia y 1.500 $\mathrm{mg} /$ día en la segunda recurrencia) ${ }^{16-19}$ (tabla 1).

Para comprobar la estabilidad de los resultados y la consistencia de las estima- ciones efectuadas en el caso base del estudio (realizado con los valores medios de todas las variables ${ }^{23}$, se hicieron análisis de sensibilidad determinísticos univariantes aplicando los valores mínimos y máximos indicados en la tabla 1 .

\section{RESULTADOS}

Se estimó que anualmente se producen 7.601 episodios de DACD en España, generándose 4.626 tratamientos con metronidazol y 2.975 tratamientos con vancomicina (considerada tanto la infección inicial como la primera y segunda recurrencia), para una incidencia media de 17,1 episodios anuales por cada 10.000 altas hospitalarias. En este supuesto, el gasto anual para el SNS se estimó en $32.157 .093 €$ (que oscilaría entre 22,9 y 45,1 millones de euros para las incidencias mínima y máxima, respectivamente) (tabla 2) . El coste por episodio de DACD es de $3.901 €$ (infección inicial), $4.875 €$ (primera recurrencia) y $5.916 €$ (segunda recurrencia). Después del tratamiento inicial, considerando la incidencia media, se producirían 1.526 casos de primera recurrencia y 505 con una segunda recurrencia, con un coste total por recurrencias de 10.426.750 $€$ anuales. El 95,6\% del gasto total de la DACD se debería a la prolongación de la duración de las estancias hospitalarias; el $0,5 \%$ a los tratamientos antibióticos; el 2,8\% a las intervenciones quirúrgicas y el $1,1 \%$ a las medidas de control de la infección (guantes, batas y termómetros desechables). A nivel autonómico, el coste de la enfermedad oscilaría entre 227.213 € y 5,6 millones de euros anuales en La Rioja y Cataluña, respectivamente (tabla 3 ). Los resultados del estudio son sensibles a las siguientes variables: incidencia y tasa de letalidad de la DACD en España y a la duración de la prolongación de la duración de la estancia hospitalaria debida a la DACD (tabla 4). 
Tabla 2

Coste anual para el Sistema Nacional de Salud de la DACD. Caso base

\begin{tabular}{|c|c|c|c|}
\hline & $\begin{array}{l}\text { Metronidazol } \\
\text { oral }\end{array}$ & $\begin{array}{c}\text { Vancomicina } \\
\text { oral }\end{array}$ & Total \\
\hline Número total de pacientes & 4.626 & 2.975 & 7.601 \\
\hline \multicolumn{4}{|l|}{ Infecciones iniciales } \\
\hline Costes hospitalarios & $14.111 .470 €$ & $6.640 .692 €$ & $20.752 .162 €$ \\
\hline Costes farmacológicos & $12.271 €$ & $61.490 €$ & $73.762 €$ \\
\hline Coste medio de la cirugía & $448.532 €$ & $211.074 €$ & $659.606 €$ \\
\hline Coste medio medidas de control & $166.473 €$ & $78.340 €$ & $244.814 €$ \\
\hline \multicolumn{4}{|l|}{$1^{a}$ recurrencia } \\
\hline Número de primeras recurrencias & 1.098 & 428 & 1.526 \\
\hline Costes hospitalarios & $3.137 .773 €$ & $3.993 .530 €$ & $7.131 .303 €$ \\
\hline Costes farmacológicos & $2.176 €$ & $58.969 €$ & $61.145 €$ \\
\hline Coste medio de la cirugía & $79.522 €$ & $101.210 €$ & $180.732 €$ \\
\hline Coste medio medidas de control & $29.515 €$ & $37.564 €$ & $67.079 €$ \\
\hline \multicolumn{4}{|l|}{$2^{a}$ recurrencia } \\
\hline Número de segundas recurrencias & 202 & 303 & 505 \\
\hline Costes hospitalarios & $946.759 €$ & $1.922 .208 €$ & $2.868 .967 €$ \\
\hline Costes farmacológicos & $540 €$ & $35.008 €$ & $35.548 €$ \\
\hline Coste medio de la cirugía & $19.729 €$ & $40.057 €$ & $59.786 €$ \\
\hline Coste medio medidas de control & $7.323 €$ & $14.867 €$ & $22.190 €$ \\
\hline \multicolumn{4}{|c|}{ Procedimiento total (infección inicial y recurrencias) } \\
\hline Costes hospitalarios & $18.196 .003 €$ & $12.556 .430 €$ & $30.752 .432 €$ \\
\hline Costes farmacológicos & $14.987 €$ & $155.468 €$ & $170.454 €$ \\
\hline Coste medio de la cirugía & $547.783 €$ & $352.340 €$ & $900.124 €$ \\
\hline Coste medio medidas de control & $203.311 €$ & $130.772 €$ & $334.082 €$ \\
\hline \multicolumn{3}{|c|}{ Coste del procedimiento total (infección inicial y recurrencias) } & $32.157 .093 €$ \\
\hline
\end{tabular}

DACD: diarrea asociada a Clostridium difficile. * Incluye el número de casos (no de pacientes) tratados tanto en primera línea, como en primera y segunda recurrencia. El coste de la recurrencia se estima en 10.426.750€. 
Tabla 3

Coste anual para el Sistema Nacional de Salud de la DACD. Resultados por Comunidades Autónomas. Caso base

\begin{tabular}{|l|c|c|}
\hline Comunidad Autónoma & $\begin{array}{c}\text { No estimado de } \\
\text { episodios anuales de } \\
\text { DACD }\end{array}$ & $\begin{array}{c}\text { Coste anual } \\
\text { de la DACD } \\
€\end{array}$ \\
\hline Andalucía & 1.123 & 4.750 .580 \\
\hline Aragón & 249 & 1.053 .888 \\
\hline Asturias (Principado de) & 197 & 834.598 \\
\hline Baleares (Islas) & 210 & 886.711 \\
\hline Canarias & 292 & 1.234 .158 \\
\hline Cantabria & 91 & 384.342 \\
\hline Castilla y León & 445 & 1.883 .391 \\
\hline Castilla-La Mancha & 300 & 1.269 .657 \\
\hline Cataluña & 1.331 & 5.629 .199 \\
\hline Comunidad Valenciana & 812 & 3.434 .335 \\
\hline Extremadura & 180 & 762.580 \\
\hline Galicia & 468 & 1.978 .829 \\
\hline Madrid (Comunidad de) & 1.086 & 4.595 .161 \\
\hline Murcia (Región de) & 232 & 982.783 \\
\hline Navarra (Comunidad Foral de) & 120 & 508.623 \\
\hline País Vasco & 390 & 1.649 .918 \\
\hline Rioja (La) & 54 & 227.213 \\
\hline
\end{tabular}

DACD: diarrea asociada a Clostridium difficile.

\section{Tabla 4}

Coste anual para el Sistema Nacional de Salud del tratamiento de la DACD. Resultados nacionales, en millones de euros.

\begin{tabular}{|c|c|c|}
\hline $\begin{array}{l}\text { Variable } \\
\text { (valor del caso base) }\end{array}$ & Valores & $\begin{array}{c}\text { Coste anual } \\
\text { (millones de } € \text { ) }\end{array}$ \\
\hline Caso base & Medios & 32,1 \\
\hline \multicolumn{3}{|l|}{ Análisis de sensibilidad } \\
\hline \multirow{2}{*}{ Incidencia anual de la DACD (por 10.000 altas) $: 17,1$} & 12,2 & 22,9 \\
\hline & 24,0 & 45,1 \\
\hline \multirow{2}{*}{ Tasa de letalidad en los pacientes con DACD $: 31,00 \%$} & $12,30 \%$ & 40,8 \\
\hline & $48,00 \%$ & 24,2 \\
\hline \multirow{2}{*}{$\begin{array}{l}\mathrm{N}^{\mathrm{o}} \text { de días de ingreso (infección inicial/ } \\
1^{\mathrm{a}} \text { recurrencia/ } 2^{\mathrm{a}} \text { recurrencia) }(7,4 / 9,1 / 10,8 \text { días })\end{array}$} & $3,7 / 5,0 / 7,3$ & 17,5 \\
\hline & $8,0 / 9,7 / 11,3$ & 34,3 \\
\hline \multirow{2}{*}{ Coste de un día de ingreso hospitalario: $434,00 €$} & $390,60 €$ & 29,8 \\
\hline & $477,40 €$ & 34,5 \\
\hline \multirow{2}{*}{ Coste de un día de ingreso en UCI: $1.131,07 €$} & $1.017,96 €$ & 31,4 \\
\hline & $1.244,18 €$ & 32,9 \\
\hline \multirow{2}{*}{$\begin{array}{l}\text { Ingreso en UCI (infección inicial/ } \\
1^{\mathrm{a}} \text { recurrencia/2a recurrencia) }(9,6 / 11,7 / 13,1 \%)\end{array}$} & $0,3 / 1,0 / 1,0 \%$ & 27,9 \\
\hline & $11,0 / 13,3 / 15,0 \%$ & 32,8 \\
\hline \multirow{2}{*}{ Coste de la intervención quirúrgica (GRD 148 y 149): 9.868,83€ } & $7.561,89 €$ & 31,9 \\
\hline & $12.175,78 €$ & 32,4 \\
\hline \multirow{2}{*}{$\begin{array}{l}\text { Pacientes que son intervenidos quirúrgicamente } \\
\text { (GRD } 148 \text { y 149) }(1,2 \%)\end{array}$} & $0,30 \%$ & 31,5 \\
\hline & $1,30 \%$ & 32,2 \\
\hline \multirow{2}{*}{$\begin{array}{l}\text { Medidas de control de la infección* } \\
\text { (valores medios del uso de recursos y costes unitarios) }\end{array}$} & Valores mínimos & 31,8 \\
\hline & Valores máximos & 32,5 \\
\hline
\end{tabular}

DACD: diarrea asociada a Clostridium difficile.DACD: UCI: unidad de cuidados intensivos.

* Guantes, batas y termómetros desechables. 


\section{DISCUSIÓN}

De acuerdo con el presente estudio, el gasto asociado a la DACD se debe fundamentalmente a la prolongación de la duración de la estancia hospitalaria, y asciende a 32,1 millones de euros en el conjunto del SNS.

La estimación de los actuales tratamientos antibióticos y del uso de recursos sanitarios en los pacientes con DACD se realizó mediante un panel Delphi de 3 expertos clínicos con una amplia experiencia en el manejo de la DACD, no a partir de un estudio de campo. No obstante, estos resultados son consistentes, dado que los análisis de sensibilidad muestran resultados generalmente estables para el conjunto del SNS, con excepción de las variables de incidencia, tasa de letalidad y número de días de ingreso hospitalario, cuya variación modifica sensiblemente los resultados del análisis.

Es importante destacar que los valores extremos de incidencia anual de la DACD en España obtenidos en diferentes estudios ${ }^{4,5}$ determinan significativamente el coste estimado de la enfermedad en nuestro país. A este respecto, debe tenerse en cuenta que la elevada incidencia obtenida mediante la detección sistemática de Clostridium difficile pone de manifiesto la existencia de un posible infradiagnóstico de este microorganismo en los casos de diarrea adquirida en el ámbito hospitalario ${ }^{5}$.

Aunque se dispone de varios estudios que estiman el uso de recursos en los pacientes con DACD, no distinguen los costes exclusivos de la DACD ${ }^{6,9}$. Solo se ha identificado un estudio que intenta estimar la carga de la DACD en España ${ }^{10}$. Se trata de una revisión bibliográfica realizada para varios países europeos de la que únicamente se dispone de resultados resumidos. Según esta revisión, la duración de la estancia hospitalaria por la
DACD sería de 18 días en España. En el presente estudio la duración máxima estimada fue menor: 8,0; 9,7 y 11,3 días en el caso de la infección inicial, la primera y la segunda recurrencia respectivamente. Esta diferencia podría deberse al hecho de que en nuestro estudio se consideró exclusivamente la prolongación de la estancia debida a la DACD, no la estancia completa de un paciente ingresado por otras causas y que padece una DACD nosocomial.

Recientemente se ha comercializado la Fidaxomicina, una nueva clase de antibiótico macrólido, aproximadamente 8 veces más activo in vitro que la Vancomicina frente a las cepas de Clostridium difficile, con una mínima absorción sistémica, que alcanza altas concentraciones fecales ${ }^{24}$. Este antibiótico no se incluyó en el presente estudio debido a que se comercializó con posterioridad a la realización del presente trabajo.

Sería de interés efectuar un estudio observacional que permita estimar en pacientes españoles la carga de la DACD. Mientras tanto, puede concluirse que el coste de la enfermedad se debe principalmente a la prolongación de la duración de la estancia hospitalaria y tiene un gran impacto económico en el SNS.

\section{BIBLIOGRAFÍA}

1. Bujanda L, Cosme A. Diarrea asociada a Clostridium difficile. Gastroenterol Hepatol. 2009; 32: 48-56.

2. Asensio A, Monge D. Epidemiología de la infección por Clostridium difficile en España Enferm Infecc Microbiol Clin. 2011; 30: 333-7.

3. Alcalá L, Marín M, Martín A, Sánchez-Somolinos M, Catalán P, Peláez MT, et al. On behalf of the Spanish Clostridium difficile Study Group. Laboratory diagnosis of Clostridium difficile infection in Spain: a population-based survey. J Hosp Infect. 2011; 79: 13-7. 
4. Asensio A, Vaque-Rafart J, Calbo-Torrecillas F, Gestal-Otero JJ, López-Fernández F, Trila-García A, et al, EPINE Working Group. Increasing rates in Clostridium difficile infection (CDI) among hospitalised patients, Spain 1999-2007. Eurosruveillance. 2008; 13: 1-4.

5. Alcalá L, Martín A, Marín M, Sánchez-Somolinos M, Catalán P, Peláez T, Bouza E; Spanish Clostridium difficile Study Group. The undiagnosed cases of Clostridium difficile infection in a whole nation: where is the problem? Clin Microbiol Infect. 2012 Jul;18(7):E204-13. doi: 10.1111/j.1469-0691.2012.03883.x. Epub 2012 May 7.

6. Monge D, Morosini M, Millán I, Pérez Canosa C, Manso M, Guzman MF, et al. Factores de riesgo de infección por Clostridium difficile en pacientes hospitalizados. Med Clin (Barc). 2011;137:575-80.

7. Vardakas KZ, Polyzos KA, Patouni K, Rafailidis PI, Samonis G, Falagas ME. Treatment failure and recurrence of Clostridium difficile infection following treatment with vancomycin or metronidazole: a systematic review of the evidence. Int J Antimicrob Agents. 2012; 40: 1-8.

8. Aslam S, Hamill RJ, Musher DM. Treatment of Clostridium difficile-associated disease: old therapies and new strategies. Lancet Infect Dis. 2005; 5: 549-57.

9. Pareja T, Hornillos M. Factores epidemiológicos, clínicos y analíticos asociados la diarrea por Clostridium difficile en población anciana hospitalizada. Estudio de casos y controles. Rev Esp Geriatr Gerontol. 2007; 42: 257-62.

10 .Wiegand P, Nathwani D, Wilcox M, Stephens JM, Wan W, Shelbaya A, et al. Clinical and economic burden of hospital onset health care facility acquired Clostridium difficile infection (HO-HCFA-CDI) in Europe: a systematic review. Value in Health. 2011; 14: A267 (PIN 10).

11. Instituto Nacional de Estadística. Encuesta de morbilidad hospitalaria 2010 . Disponible en: http://www.ine.es (consulta: 25 de marzo de 2012).

12. Crook D, et al. Randomized clinical trial (RCT) in Clostridium difficile infection (CDI) confirms equivalent cure rate and lower recurrence rate of fidaxomicin (FDX) versus vancomycin (VCN). 20th Annual European Congress of Clinical Microbiology and Infectious Diseases (ECCMID); Vienna, Austria, 10-13 April 2010; Abstract LB2401.

13. Wenisch C, Parschalk B, Hasenhündl M, Hirschl AM, Graninger W. Comparison of vancomycin, teicoplanin, metronidazole, and fusidic acid for the treatment of Clostridium difficile-associated diarrhea. Clin Infect Dis. 1996; 22: 813-8.
14. Cornely O, Miller M, Louie T, et al. Randomized controlled trial (RCT) of fidaxomicin (FDX) versus vancomycin (VAN) in treatment of recurrent Clostridium difficile infection (CDI). 50th Interscience Conference on Antimicrobial Agents and Chemotherapy (ICAAC) 2010; Abstract L1-1305.

15. Musher D et al. Relatively Poor Outcome after Treatment of Clostridium difficile Colitis with Metronidazole. Clin Infect Dis. 2005; 40:1586-90.

16. Base de datos de costes sanitarios en España. Versión 4.2. Madrid: Health Value, 2012. Accesible en DVD (consulta: 30 de abril de 2012).

17. Flagyl $250 \mathrm{mg}$ comprimidos. Ficha técnica. Disponible en: http://www.aemps.gob.es/cima/especialidad.do?meto$\mathrm{do}=$ verFichaWordPdf $\&$ codigo $=35034 \&$ formato $=$ pdf $\&$ for mulario=FICHAS\&file=ficha.pdf (consulta: 25 de marzo de 2012).

18. Vancomicina Hospira $500 \mathrm{mg}$, polvo para solución inyectable. Disponible en URL: http://www.aemps.gob.es/cima/especialidad.do?metodo $=\mathrm{v}$ erFichaWordPdf $\&$ codigo $=62520 \&$ formato $=$ pdf $\&$ formulario=FICHAS\&file=ficha.pdf (consulta: 25 de marzo de 2012).

19. Base de datos de medicamentos. Consejo General de Colegios Oficiales de Farmacéuticos. Disponible en: http://www.portalfarma.com/home.nsf (consulta: $30 \mathrm{de}$ abril de 2011).

20. Hospital Universitario Fundación Alcorcón. PA 01/10. Suministro de guantes desechables: Disponible en: http://javascript:abrirUrl('/cs/Satellite?blobcol=urldata\&bl obheader $=$ application $\% 2 \mathrm{~F}$ pdf\&blobheadername $1=$ Content-disposition\&blobheadername $2=$ cadena\&blobheadervalue $1=$ filename $\% 3$ DLicitaci $\% C 3 \% B 3 n+D O U E+01-$ 10 . p d f \& b 1 o b h e a d e r v a 1 u e $2=$ language $\% 3$ Des $\% 26$ site $\% 3 \mathrm{DHospitalFundacionHospita-}$ 1Alcorcon\&blobkey=id\&blobtable=MungoBlobs\&blobwhere $=1220687545275 \&$ ssbinary $=$ true') $($ consulta: 31 de mayo de 2012).

21. Resolución de adjudicación de la Dirección Gerencia del Servicio Andaluz de Salud de 17 de septiembre de 2009 para el suministro de mascarillas de quirófano y batas desechables no estériles para la pandemia de gripe A, C.C./PN 4003/2009. Disponible en: http://www.juntadeandalucia.es/ contratacion/document/download? refCode $=2009$ 0000011367\&refDoc $=2009-0000011367-0$ (consulta: 31 de mayo de 2012).

22. Suministros médicos MEVESUR. Termómetro desechable TEMPA-DOT. Caja de 100 unidades. Disponible en: http://www.mevesur.com/termometro-desechable-tempa-caja-unidades-p-3316.html (consulta: 31 de mayo de 2012). 
23. Rubio-Terrés C, Cobo E, Sacristán JA, Prieto L, del Llano J, Badia X, por el Grupo ECOMED. Análisis de la incertidumbre en las evaluaciones económicas de intervenciones sanitarias. Med Clín (Barc). 2004; 122: 668674.

24. Golan Y, Epstein L. Safety and efficacy of fidaxomicin in the treatment of Clostridium difficile-associated diarrhea. Therap Adv Gastroenterol. 2012; 5: 395-402. 